Modular Sampling and Analysis

Techniques for the Real-Time Analysis of Human Breath

Matthias Frank, George Farquar, Krisl Adams, Michael Bogan, Audrey Martin, Hennry Benner, Christopher Spadaccini, Paul Steele, Cristina Davis, Bryan Loyola, Josh Morgan, Shankar Sankaran

July 10, 2007

Sensors Expo 2007

Rosemont, IL, United States

June 11, 2007 through June 13, 2007 
This document was prepared as an account of work sponsored by an agency of the United States Government. Neither the United States Government nor the University of California nor any of their employees, makes any warranty, express or implied, or assumes any legal liability or responsibility for the accuracy, completeness, or usefulness of any information, apparatus, product, or process disclosed, or represents that its use would not infringe privately owned rights. Reference herein to any specific commercial product, process, or service by trade name, trademark, manufacturer, or otherwise, does not necessarily constitute or imply its endorsement, recommendation, or favoring by the United States Government or the University of California. The views and opinions of authors expressed herein do not necessarily state or reflect those of the United States Government or the University of California, and shall not be used for advertising or product endorsement purposes. 


\title{
Modular Sampling and Analysis Techniques for the Real-Time Analysis of Human Breath
}

\author{
Lawrence Livermore National Laboratory, \\ Physics Directorate, 7000 East Avenue, L-211, Livermore \\ CA 94551, USA, frank1@1lnl.gov \\ Shankar Sankaran, Bryan Loyola, Josh Morgan, Cristina Davis \\ University of California Davis, \\ Bainer Hall, One Shields Avenue, Davis, CA 95616, USA
}

Matthias Frank, George Farquar, Kristl Adams, Michael Bogan, Audrey Martin, Henry Benner, Christopher Spadaccini, Paul Steele

\begin{abstract}
At LLNL and UC Davis, we are developing several techniques for the real-time sampling and analysis of trace gases, aerosols and exhaled breath that could be useful for a modular, integrated system for breath analysis. Those techniques include single-particle bioaerosol mass spectrometry (BAMS) for the analysis of exhaled aerosol particles or droplets as well as breath samplers integrated with gas chromatography mass spectrometry (GC-MS) or MEMSbased differential mobility spectrometry (DMS). We describe these techniques and present recent data obtained from human breath or breath condensate, in particular, addressing the question of how environmental exposure influences the composition of breath.
\end{abstract}

\section{INTRODUCTION}

In recent years, it has been shown that volatile organic compounds (VOCs) exhaled in breath have the potential to be used as bio-markers for the physiological state of a person, systemic diseases (such as cancers and ulcers) as well as respiratory diseases $[1,2]$. As a result breath tests are being used for early detection of heart transplant rejection [3], stomach ulcers [4], and breast cancer [5]. Nevertheless, clinical research on breath analysis is still in its early stages, in part, due to limitations in breath analysis instrumentation and detector technology. Besides rapid, early medical diagnostics, breath VOC analysis may also be useful to assess environmental exposure of individuals to solvents and other chemicals [6,7]. Currently explored technologies for breath analysis include gas chromatography mass spectrometry (GC-MS), ion mobility spectrometry (IMS) and differential mobility spectrometry (DMS).

Aerosol droplets exhaled by human subjects may carry tell-tale signatures of respiratory diseases in particular when entire intact bacteria or virus are exhaled or coughedout in human expirations or sputum. Single particle bioaerosol mass spectrometry (BAMS) is applied to human expirations and breath analysis where early data suggests respiratory infections such as tuberculosis may be detected in near real-time analysis of expired human sputum and possibly breath.

In addition to recent instrument technology advances, there is a need for future work in signature and bio-marker development as well as exploring the correlations between disease and environmental exposure of a subject and their breath composition (both the VOC and aerosol droplet phase). Instrument advances will see the development of smaller, more sensitive and deployable breath analysis systems that can ideally analyze breath in or near real-time.

\section{METHODS}

\section{A. Bioaerosol Mass Spectrometry (BAMS) for Aerosol Particle Analysis}

Single aerosol particle bioaerosol mass spectrometry systems have been developed at LLNL for biodefense applications over the last decade and are now being applied to the rapid analysis of cells or cell fragments in human effluents, such as exhaled aerosols, and screening of those effluents for pathogens. The BAMS technique allows analyzing the biochemical composition of single aerosol particles and cells in real-time and, in some cases, provides species discrimination between different species of bacteria [8-10]. As shown schematically in Fig. 1, aerosol particles are sampled either directly from air or an aerosol generator into the inlet of a BAMS system. Particles are aerodynamically directed into a tightly focused particle beam by means of an aerodynamic lens that also introduces the particles into the vacuum of the BAMS mass spectrometer. Following the focusing lens and vacuum introduction, individual particles are aerodynamically sized by measuring their speed using light scattering off several continuous wave laser beams. Next, the particles' fluorescence properties are probed by exciting them with a pulsed, low power UV laser. When particles reach the ion source of the mass spectrometer they are hit by a powerful desorption and ionization laser 
pulse and characteristic molecular ions (both positive and negative) are measured by time-of-flight mass spectrometry in a bipolar mass spectrometer. The whole analysis process takes a fraction of a second per particle. Thus tens of particles can be analyzed per second. Little to no sample preparation is necessary.

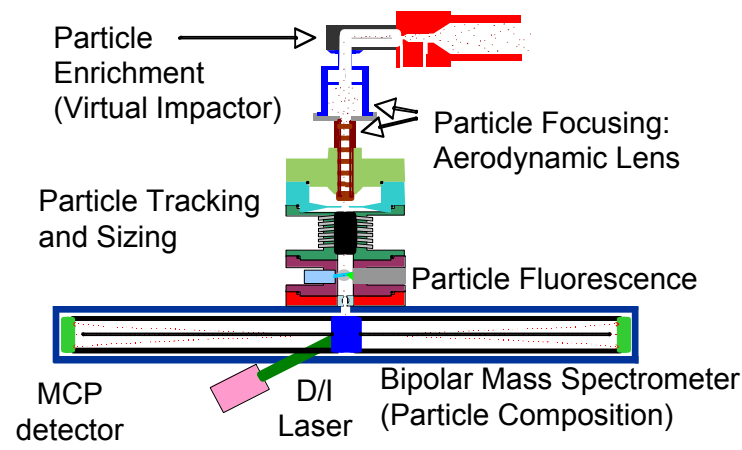

Fig. 1: Schematic of a LLNL BAMS system.

Using a LLNL BAMS system, we have analyzed micrometer sized aerosol droplets comprised of pure bovine lung surfactant, water droplets containing single cells of Mycobacterium smegmatis (a harmless simulant for $M$. tuberculosis) and droplets of lung surfactant containing single cells of $M$. smegmatis.

\section{B. Sample preparation for BAMS analysis}

Survanta brand bovine lung surfactant (LS) was obtained from Abbott Laboratories and diluted 1:10 in deionized water before aerosolization. Mycobacterium smegmatis (ATCC \#19420) was grown in Middlebrook 7H9 broth (BD 271310) supplemented with $0.2 \%$ glycerol and $10 \%$ albumin-dextrose-catalase (ADC) enrichment (BD 212352). Cultures were incubated at $37^{\circ} \mathrm{C}$ on a platform shaker $(150$ rpm) until mid to late log phase and then collected by centrifugation. Water-soluble media components were removed by washing the pellet $\mathrm{x} 3$ with sterile water. The washed pellet was subsequently re-suspended in water to a final concentration of $10^{9}-10^{10}$ cells $/ \mathrm{mL}$ and refrigerated until mass spectral analysis. Aliquots of the prepared $M$. smegmatis were analyzed within 24 hours of the postculturing treatment.

All liquid suspension samples (lung surfactant - LS, $M$. smegmatis and a mixture of lung surfactant and $M$. smegmatis - LS + M. smeg.) were introduced into the BAMS system by aerosolization in a stream of nitrogen gas (flow rate $\sim 1.5 \mathrm{~L} / \mathrm{min}$ ) using a low volume disposable nebulizer (Salter Labs, Arvin, CA, USA). Aerosol droplets were directed through an in-line diffusion dryer containing a silica desiccant resulting in particles with average aerodynamic diameter in the range of $0.5-2.5 \mu \mathrm{m}$ before introduction into the mass spectrometer.

\section{GC-MS for breath condensate analysis}

GC-MS is being used at LLNL for the analysis of respiratory effluents. GC-MS is a promising technique because of its sensitivity, specificity, and the orthogonal data it provides. Briefly, a sample is introduced to the injection point, and is swept through the analytical column by a carrier gas, typically helium. The analytes are separated in time as a temperature ramp is induced on the column. The samples are then transferred to the mass spectrometer, where spectra are obtained for each eluent. Using a commercial GC-MS system (Agilent 6890A, 5973N) we have conducted a series of experiments to explore how this technique can be used to assess environmental exposure of an individual by analyzing breath or breath condensate.

\section{Sample preparation for breath condensate analysis}

Breath condensate was obtained at each time point by breathing into an R-Tube ${ }^{\mathrm{TM}}$ breath collection device (Respiratory Research, Inc.) with a condenser cooled to $-80^{\circ}$ $\mathrm{C}$ for 15 minutes (resulting in $\sim 1 \mathrm{~mL}$ of condensate) and transferring $500 \mu \mathrm{L}$ aliquots to plastic centrifuge tubes. Because of the low concentrations of target compounds in breath (nanomolar or lower) [11], solid-phase microextraction (SPME) was used to preconcentrate samples. A $65 \mu \mathrm{m}$ PDMS/DVB Stableflex SPME fiber (Sigma-Aldrich, Inc.) was submerged in an aliquot of breath condensate for 10 minutes. The fiber was then placed in the injection port of the GC-MS where the sample was thermally desorbed from the fiber and subjected to a 17 minute temperature program for analysis.

\section{RESULTS}

\section{A. BAMS Results}

The BAMS experiments show that the characteristic mass spectral markers observed with BAMS on pure Mycobacteria are largely preserved when these bacteria are embedded in a complex matrix (bovine lung surfactant) and can be used to detect the presence of these bacteria within lung surfactant. Fig. 2 shows results obtained with Mycobacterium smegmatis, a non-virulent surrogate for $M$. tuberculosis. The three figures depict various $\mathrm{m} / \mathrm{z}$ ranges from the mass spectra measured from pure lung surfactant (LS, blue trace), M. smegmatis embedded in LS (black trace) and pure $M$. smegmatis in water (red trace). Note that negative $\mathrm{m} / \mathrm{z}$ values correspond to the mass spectra from negative ions (BAMS measures positive and negative ions simultaneously by recording the positive and negative ion mass spectra in separate channels).

The data show that characteristic mass spectral peaks observed with pure $M$. smegmatis are conserved when $M$. smegmatis is embedded in lung surfactant and that those peaks are clearly distinguishable from the background of lung surfactant. Both the positive and negative $\mathrm{m} / \mathrm{z}$ spectrum show an easy distinction between lung surfactant (having little ion amplitude) and the stronger mycobacterium ion 
signal. We suggest that the -421 peak is tentatively due to the deprotonated disaccharide, trehalose-2-sulfate ion, which is a precursor for the sulfolipid virulence factor known as sulfolipid 1 (SL-1) [12-14]. SL-1 has been positively identified as a component of the virulence factor in M. smegmatis and M. tuberculosis H37Ra [15]. These data indicate the potential of a BAMS system for detecting tuberculosis and other human respiratory diseases within the complex background of exhaled human aerosols. Similar experiments using Mycobacteria and other microorganisms embedded in human breath condensate or sputum are in progress and will help to determine whether BAMS could be useful in rapidly diagnosing certain respiratory diseases, e.g., tuberculosis.
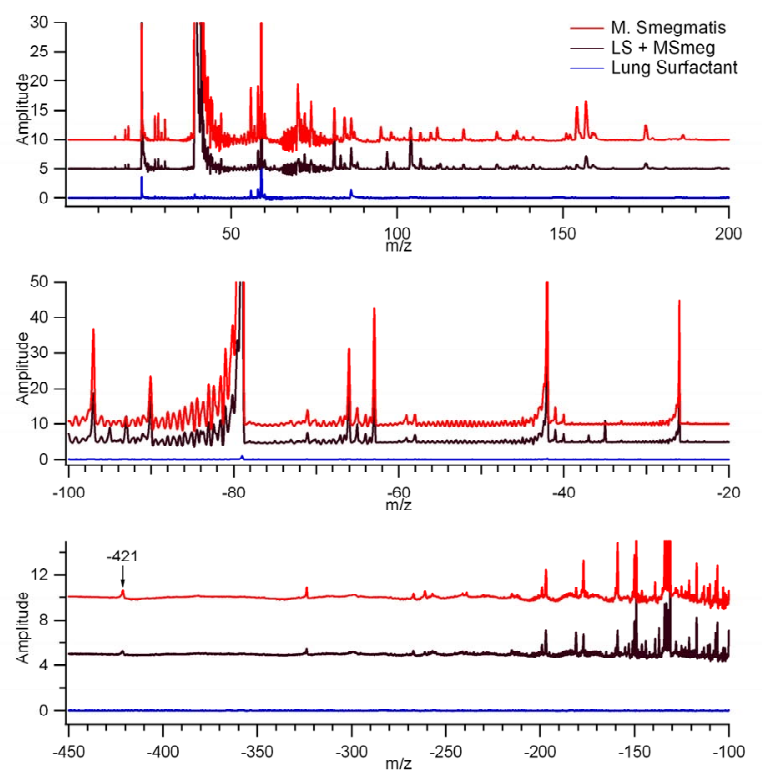

Fig. 2: Mycobacterium smegmatis detection in a background of bovine lung surfactant with bioaerosol mass spectrometry (BAMS). The three graphs show different $\mathrm{m} / \mathrm{z}$ ranges. Average m/z spectra are shown: $\sim 900$ particles each of $M$. smeg and lung surfactant, respectively, and $\sim 600$ particles of M. smeg. embedded in lung surfactant. The peak at $\mathrm{m} / \mathrm{z}=-421$ is tentatively assigned as the deprotonated disaccharide, trehalose-2-sulfate ion which is a precursor for the SL-1 virulence factor found in both $M$. smegmatis and $M$. tuberculosis.

\section{B. GC-MS Results}

Using a commercial GC-MS system we have conducted a series of experiments to explore how this technique can be used to assess environmental exposure of an individual by analyzing breath or breath condensate. In a preliminary study, a subject was asked to donate a breath sample immediately before visiting a nail care salon where red nail polish was applied. The subject was then asked to donate breath samples after leaving the salon. Fig. 3 shows the results of GC-MS measurements of breath condensates sampled before the visit to a nail care salon and application of nail polish, 0.75 hours after the salon visit and 3.25 hours after. Note the consistency in all peaks in the spectra before time $=7.6 \mathrm{~min}$, which has been tentatively identified as camphor. The data indicate a clearly elevated level of camphor (arrow) after the salon visit and a slow decay of the camphor signal some hours after. Although some of the camphor in the breath condensate data shown below is thought to be due to inhalation of residual chemicals from the polished nails, further studies have shown that a significant camphor signal is still detected when the inhaled air source is moved further away from the polish nails.

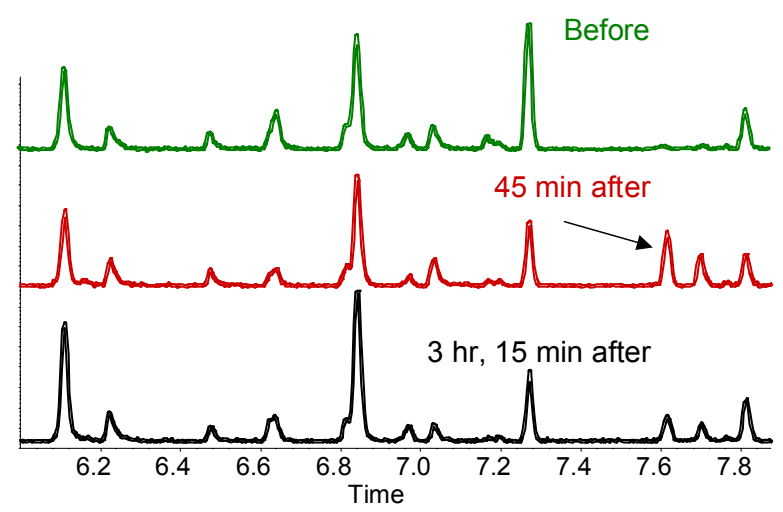

Fig. 3: Breath condensate sample extracted with a SPME fiber for 10 minutes and run with a GC-MS. All three traces are from the same subject. The top trace sample was obtained before having a manicure with red nail polish in a nail salon, the two other traces were obtained at 0.75 hours and 3.25 hours after the salon visit, respectively. Note the new compound at 7.6 min (arrow). This compound has been identified as camphor, a known environmental hazard in nail salons.

\section{CONCLUSIONS}

We have performed single-particle Bioaerosol Mass Spectrometry measurements on mycobacteria and mycobacteria embedded in bovine lung surfactant. Our results indicate that the distinct mass spectral signatures observed from pure mycobacteria are largely preserved and easily detectable by BAMS when the bacteria are embedded in the complex matrix of lung surfactant. Further measurements with BAMS that will include M. tuberculosis $\mathrm{H} 37 \mathrm{Ra}$ (an avirulent strain) are in progress and will help to evaluate the potential use of BAMS in real-time biomedical diagnostics for respiratory diseases such as tuberculosis.

We also have performed measurements on exhaled human breath condensate using GC-MS analysis to 
investigate whether trace VOCs in human breath can be used to detect environmental exposure of a person to certain chemicals some time after the exposure. Our initial results are encouraging and indicate that markers for environmental exposure may exist for days after the exposure.

\section{ACKNOWLEDGMENT}

This work was performed under the auspices of the U.S. Department of Energy by University of California Lawrence Livermore National Laboratory (LLNL) under contract W7405-ENG-48 and supported by LLNL through Laboratory Directed Research and Development (LDRD) grant no. 05ERD-053 and by a DARPA/MTO seedling project (PM Dennis Polla).

\section{REFERENCES}

[1] M. Phillips, "Breath Tests in Medicine," Scientific American, vol. 267, pp. 74-79, Jul 1992.

[2] M. Phillips and J. Greenberg, "Ion-Trap Detection of Volatile Organic-Compounds in Alveolar Breath," Clinical Chemistry, vol. 38, pp. 60-65, Jan 1992.

[3] M. Phillips, J. P. Boehmer, R. N. Cataneo, T. Cheema, H. J. Eisen, J. T. Fallon, P. E. Fisher, A. Gass, J. Greenberg, J. Kobashigawa, D. Mancini, B. Rayburn, and M. J. Zucker, "Prediction of heart transplant rejection with a breath test for markers of oxidative stress," American Journal of Cardiology, vol. 94, pp. 1593-1594, Dec 15 2004.

[4] FDA, "ALIMENTERICS PYLORI-CHEK BREATH TEST KIT."

[5] M. Phillips, R. N. Cataneo, B. A. Ditkoff, P. Fisher, J. Greenberg, R. Gunawardena, C. S. Kwon, O. Tietje, and C. Wong, "Prediction of breast cancer using volatile biomarkers in the breath," Breast Cancer Research and Treatment, vol. 99, pp. 19-21, Sep 2006.

[6] W. Q. Cao and Y. X. Duan, "Current status of methods and techniques for breath analysis," Critical Reviews in Analytical Chemistry, vol. 37, pp. 3-13, 2007.
[7] L.C.A. Amorim and Z. de L. Cardeal, "Breath analysis and its use as a biomarker in biological monitoring of occupational and environmental exposure to chemical agents," J. Chromatography B, 853, pp.1-9, 2007.

[8] D.P. Fergenson, M.E. Pitesky, H.J. Tobias, P.T. Steele, G.A. Czerwieniec, S.C. Russell, C.B. Lebrilla, J.M. Horn, K.R. Coffee, A. Srivastava, S.P. Pillai, M.P. Shih, H.L. Hall, A.J. Ramponi, J.T. Chang, R.G. Langlois, P.L. Estacio, R.T. Hadley, M. Frank and E.E. Gard, "Reagentless Detection and Classification of Individual Bioaerosol Particles in Seconds," Anal. Chem. 76, 373-378 (2004).

[9] A. Srivastava, M.E. Pitesky, P.T. Steele, H.J. Tobias, D.P. Fergenson, J.M. Horn, S.C. Russell, G.A. Czerwieniec, C.B. Lebrilla, E.E. Gard, and M. Frank, "Comprehensive Assignment of Mass Spectral Signatures from Individual Bacillus atrophaeus Spores in Matrix-Free Laser Desorption/Ionization Bioaerosol Mass Spectrometry," Anal. Chem. 77, 3315-3323 (2005).

[10] H.J. Tobias, M.P. Schafer, M. Pitesky, D.P. Fergenson, J. Horn, M. Frank, and E.E. Gard, "Bioaerosol Mass Spectrometry (BAMS) for the Rapid Detection of Individual Airborne Mycobacterium tuberculosis H37Ra Particles," Appl. Environ. Microbiol., 71, 60866095 (2005).

[11] Krotoszynski, B.K.; Gabriel, G.; O’Neill, H. J. Chromatgraph Sci. 1977, 15, 239-244.

[12] [J. D. Mougous, D. L. Akey, L. W. Riley, D. H. Lee, S. E. Munchel, C. J. Petzold, R. H. Senaratne, F. L. Lin, and M. R. Pratt, "Identification, function and structure of the mycobacterial sulfotransferase that initiates sulfolipid-1 biosynthesis," Nat Struct Mol Biol, vol. 11, pp. 721-9, 2004.

[13] J. D. Mougous, S. E. Brenner, C. R. Bertozzi, R. E. Green, and S. J. Williams, "Sulfotransferases and sulfatases in mycobacteria," Chem Biol, vol. 9, pp. 767-76, 2002.

[14] M. Jain, M. D. Leavell, J. A. Leary, J. D. Mougous, C. J. Petzold, M. W. Schelle, C. R. Bertozzi, and J. S. Cox, "Lipidomics reveals control of Mycobacterium tuberculosis virulence lipids via metabolic coupling," Proc Natl Acad Sci U S A, vol. 104, pp. 5133-8, 2007.

[15] J. D. Mougous, C. D. Leigh, J. A. Leary, S. J. Williams, M. D. Leavell, R. H. Senaratne, L. W. Riley, and C. R. Bertozzi, "Discovery of sulfated metabolites in mycobacteria with a genetic and mass spectrometric approach," Proc Natl Acad Sci US A, vol. 99, pp. 17037-42, 2002. 